\title{
Protective effect of the n-butanol Toona sinensis seed extract on diabetic nephropathy rat kidneys
}

\author{
W.Z. $\mathrm{Li}^{1}$, X.H. Wang ${ }^{1}$, H.X. Zhang' ${ }^{2}$, S.M. Mao ${ }^{3}$ and C.Z. Zhao ${ }^{3}$ \\ ${ }^{1}$ College of Pharmacy, Weifang Medical University, Weifang, China \\ ${ }^{2}$ School of Clinical Medicine, Weifang Medical University, Weifang, China \\ ${ }^{3}$ Department of Pharmacology and Applied Pharmacology Laboratory, \\ Weifang Medical University, Weifang, China \\ Corresponding author: W.Z. Li \\ E-mail: liwzhong_ı@163.com
}

Genet. Mol. Res. 15 (1): gmr.15017403

Received August 7, 2015

Accepted November 13, 2015

Published March 24, 2016

DOI http://dx.doi.org/10.4238/gmr.15017403

ABSTRACT. The objective of this study was to observe the protective effect of the n-butyl alcohol phase of Toona sinensis seed extract on the kidneys of diabetic nephropathy (DN) rats and its preliminary mechanism. Male wistar rats were administered a normal or high-fat diet for 1 month. DN rats were divided into a model group and a petroleum ether phase of $T$. sinensis seed extract intervention group. The intervention group was administered $5 \mathrm{mg} \cdot 100$ $\mathrm{g}^{-1}$. day ${ }^{-1}$ extract. After treatment for 10 weeks, the rats were sacrificed and blood samples and the renal cortex were collected. Biochemical indicators in the serum and renal indices were assessed. Pathological changes of the renal tissues were also determined. Changes in the renal structure and protein levels were detected. Compared with the normal group, the blood glucose, urinary albumin, renal index, and oxidative stress index were sharply increased in the model group. The protein levels of TGF- $\beta 1$, collagen IV, and connective tissue growth factor (CTGF) were increased. Compared with the model group, the $n$-butyl alcohol phase of $T$. sinensis seed extract significantly reduced the blood glucose, urinary albumin, renal index, oxidative stress index, serum creatinine, 
and urea nitrogen levels. The renal pathology abnormality was improved in DN rats. The protein levels of TGF- $\beta 1$, collagen IV, and CTGF were increased. The expression of TGF- $\beta 1$, collagen IV, and CTGF decreased. In conclusion, the n-butyl alcohol phase of $T$. sinensis seed extract has protective effects on DN rats via the inhibition of oxidative stress and protein expression of TGF- $\beta 1$, collagen IV, and CTGF.

Key words: N-butyl alcohol phase; Toona sinensis seed extracts; Diabetic nephropathy; Oxidative stress

\section{INTRODUCTION}

Diabetic nephropathy (DN) is glomerulosclerosis caused by the abnormal metabolism of diabetes accompanied by proteinuria, and its disease mechanism is a popular research topic (Satirapoj, 2012). Excessive production of reactive oxygen species is the common upstream event of the polyol pathway flux, protein kinase $\mathrm{C}$, advanced glycation end-products, and the hexosamine biosynthesis pathway. Oxidative stress is an independent factor in diabetes mellitus (DM) and angiopathy, and is considered a common mechanism of the disease.

Shandong is one of the main production areas of Toona sinensis seeds. T. sinensis seeds are infused in water or decocted with water to treat DN in Weifang, Linyi, and Liaocheng. T. sinensis seeds have many effects such as decreasing blood glucose, antioxidation, anticoagulation, and protective effects on neurons (Li and Chen, 2009, 2010; Xing and Chen, 2010; Jin and Chen, 2011; Du et al., 2011; Zhao et al., 2011). Here, we studied the effects of n-butyl alcohol extract (NBAE) of $T$. sinensis seeds on DN rats with oxidative stress and the roles of oxidative stress in the disease mechanism of DN.

\section{MATERIAL AND METHODS}

\section{Animals}

Wistar rats were purchased from the Medical Experimental Animals Center of Shandong Lu Kang. The certification number was SCXK Lu 20130001.

\section{Drugs and regents}

T. sinensis seeds (Jinan Shengke Technology Company) were identified as T. sinensis (A. Juss.) Roem. fruits by Dr. Xu Chongmei of the biopharmacy department of Weifang Medical Academy. The NBAE of the T. sinensis seeds was prepared by the Pharmacy Department Laboratory of Weifang Medical Academy. Streptozotocin (STZ, Sigma), detection kits (Nanjing Jiancheng Bioengineer Research Laboratory), and antibodies were purchased from Sigma-Aldrich and Santa Cruz.

\section{Instruments}

The blood glucose meter was obtained from Roche. The enzyme mark instrument was from Bio-Rad. The automated biochemical analyzer was obtained from Rili. The microscope was from Olympus. The transmission electron microscope used was a Rili (H-7650). 


\section{Methods}

\section{Model and treatment groups}

Male Wistar rats weighing 180-200 g were fed a normal diet for 1 week. Then, 10 rats were randomly selected as the control group and given normal food. Twenty-five rats were used as model rats and were administered only food with high fat and glucose (Shanghai Silaike Experimental

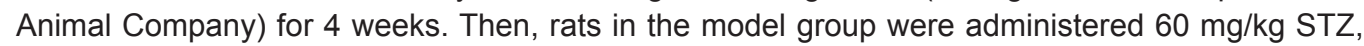
and their blood glucose levels were within 16.7-25.0 mM (Yang and Li, 1993; Tang et al., 2010; Hu et al., 2011). The rats were fed until their urine protein content was over $20 \mu \mathrm{g} / \mathrm{mL}$. Nine of the DM rats were used in the model group and 10 in the NBAE of $T$. sinensis seed group, in which rats were treated with $5 \mathrm{mg} \cdot 100 \mathrm{~g}^{-1} \cdot$ day $^{-1}$ for 10 weeks. The other groups were treated with the same amount of normal saline.

\section{Sample collection}

After the last treatment, the 24-h urine of the rats was collected, centrifuged, and stored at $4^{\circ} \mathrm{C}$. Rats were weighed and then injected with $10 \%$ chloral hydrate $(0.4 \mathrm{~mL} / 100 \mathrm{~g})$. Blood was collected, centrifuged to obtain the serum, and stored at $-20^{\circ} \mathrm{C}$. The kidneys were washed with $4^{\circ} \mathrm{C}$ normal saline via infusion from the heart until the color was white. Two kidneys were obtained and weighed after removal of the capsule. A portion was used to make an electron microscope specimen, and the other parts were fixed with paraformaldehyde.

\section{Detection of biochemical index}

The rat fasting blood glucose, serum creatinine (Scr), blood urea nitrogen (BUN), and urinary creatinine $(\mathrm{Ucr})$ were detected. Kits were used to measure urine protein, serum total antioxidant capacity (T-AOC), superoxide dismutase (SOD), malondialdehyde (MDA), glutathione peroxidase (GSH-PX), and catalase (CAT) contents.

\section{Kidney pathological parameters}

The specimens were fixed with paraformaldehyde, embedded using paraffin, sectioned, and stained using hematoxylin and eosin (H\&E), periodic acid-silver metheramine (PASM), periodic acid-Schiff (PAS), and Masson's trichrome. The pathological changes in the rat kidneys were observed under a microscope.

\section{Immunohistochemistry}

Five visual fields from each immunohistochemistry sample were imaged under 400X magnification. The I-solutions software was used to quantify and analyze the positive results, and then the average positive strength was obtained.

\section{Electron microscope observation of kidney}

Kidney specimens were fixed, stained, and observed using electron microscopy. 


\section{Statistical analysis}

SPSS 15.0 was used to analyze the data, and data are reported as means \pm SD. The quantitative data were analyzed by a test of normality, and the differences between groups were analyzed using one-way ANOVA. $\mathrm{P}<0.05$ was considered statistically significant.

\section{RESULTS}

\section{Effects of $n$-butyl alcohol phase of $T$. sinensis seed extract on the weight and serum glucose of DN rats}

The weight decreased in the DN group compared with the control group $(P<0.01)$. Additionally, the kidney index (kidney weight/body weight) and blood glucose increased $(P<0.01)$. Compared with the DN group, the weight of the NBAE group significantly increased $(P<0.05)$, and its kidney index and blood glucose decreased $(P<0.05$; Table 1$)$.

Table 1. Body weight, index of renal hypertrophy, and the renal function-related parameters in different groups at the end of treatment.

\begin{tabular}{l|c|c|c}
\hline & Normal group & Model group & NBAE group \\
\hline Body weight (BW) (g/rat) & $612.7 \pm 24.21$ & $410.5 \pm 39.12^{*}$ & $455.3 \pm 41.58^{\text {*\# }}$ \\
\hline KW/BW ratio $(\%)$ & $3.28 \pm 0.59$ & $5.02 \pm 0.71^{*}$ & $4.18 \pm 0.56^{* \#}$ \\
\hline Serum glucose $(\mathrm{mM})$ & $6.72 \pm 1.05$ & $20.91 \pm 3.87^{*}$ & $11.56 \pm 2.79^{\text {*\# }}$ \\
\hline HbAlc $(\%)$ & $4.15 \pm 0.36$ & $14.07 \pm 1.28^{*}$ & $12.55 \pm 1.05^{* \#}$ \\
\hline Urine volume $(\mathrm{mL} / 100 \mathrm{~g} / 24 \mathrm{~h})$ & $5.94 \pm 0.78$ & $34.25 \pm 7.26^{*}$ & $19.23 \pm 3.21^{\text {*\# }}$ \\
\hline Urine protein $(\mathrm{mg} / 24 \mathrm{~h})$ & $10.93 \pm 1.06$ & $28.93 \pm 2.76^{*}$ & $16.03 \pm 1.55^{\text {*\# }}$ \\
\hline Ucr $(\mathrm{mM})$ & $12.65 \pm 1.73$ & $2.86 \pm 0.49^{*}$ & $6.35 \pm 0.93^{* \#}$ \\
\hline Scr $(\mu \mathrm{M})$ & $42.58 \pm 4.16$ & $69.75 \pm 5.64^{*}$ & $57.83 \pm 5.02^{\text {*\# }}$ \\
\hline BUN $(\mathrm{mM})$ & $6.71 \pm 0.98$ & $12.26 \pm 1.35^{*}$ & $8.51 \pm 1.25^{\text {*\# }}$ \\
\hline
\end{tabular}

$\mathrm{NBAE}=\mathrm{n}$-butyl alcohol extract; $\mathrm{KW}=$ kidney weight; $\mathrm{HbAlc}=$ hemoglobin $\mathrm{A} 1 \mathrm{c} ; \mathrm{Ucr}=$ urinary creatinine; Scr = serum creatinine; $\mathrm{BUN}=$ blood urea nitrogen. ${ }^{*} \mathrm{P}<0.01$ compared to the normal group. ${ }^{\#} \mathrm{P}<0.05$ compared to the model group.

\section{Improvement in kidney function in DN rats}

Compared with the control group, the 24-h urine amount, urine protein, HbAlc, Scr, and BUN were improved in DN rats $(P<0.01)$, while the $U c r$ decreased $(P<0.01)$. Compared with the DN group, the 24-h urine amount, urine protein, $\mathrm{HbAlc}$, Scr, and BUN decreased $(P<0.01$ or 0.05$)$, while the Ucr increased $(P<0.01)$. The results are shown in Table 1.

\section{Effects of the $n$-butyl alcohol phase of $T$. sinensis seed extract on oxidative stress parameters of DN rats}

Compared with the control group, the activities of T-AOC, SOD, GSH-Px, and CAT were all decreased in the DN group $(P<0.01)$, while the MDA level improved $(P<0.01)$. Compared with the DN group, the activities of T-AOC, SOD, GSH-Px, and CAT increased in the NBAE group $(\mathrm{P}<$ 0.01 or 0.05$)$, while the MDA level decreased $(P<0.01)$. Results are shown in Table 2. 
Table 2. Antioxidant indices in experimental rats at the end of treatment.

\begin{tabular}{l|c|c|c}
\hline & Normal group & Model group & NBAE group \\
\hline T-AOC $(\mathrm{U} / \mathrm{mL})$ & $10.98 \pm 2.25$ & $5.49 \pm 1.46^{*}$ & $8.71 \pm 1.56^{* *}$ \\
\hline SOD $(\mathrm{U} / \mathrm{mL})$ & $153.46 \pm 9.35$ & $118.79 \pm 7.34^{*}$ & $139.53 \pm 8.92^{* *}$ \\
\hline MDA $(\mathrm{nM})$ & $5.51 \pm 0.98$ & $12.14 \pm 1.13^{*}$ & $7.12 \pm 0.75^{* *}$ \\
\hline GSH-Px $(\mathrm{U} / \mathrm{mL})$ & $528.17 \pm 6.95$ & $221.81 \pm 11.25^{\star}$ & $240.02 \pm 16.97^{* \#}$ \\
\hline CAT $(\mathrm{U} / \mathrm{mL})$ & $4.52 \pm 0.63$ & $1.94 \pm 0.48^{*}$ & $3.49 \pm 0.56^{* *}$ \\
\hline
\end{tabular}

NBAE $=$ n-butyl alcohol extract; T-AOC $=$ serum total antioxidant capacity; SOD $=$ superoxide dismutase, MDA = malondialdehyde, GSH-PX = glutathione peroxidase; $\mathrm{CAT}=$ catalase. ${ }^{*} \mathrm{P}<0.01$ compared to the normal group. ${ }^{\#} \mathrm{P}<$ 0.05 and ${ }^{* *} P<0.01$ compared to the model group.

\section{Improvement in pathological injury of STZ diabetic rats by oxidative stress parameters}

The pathological changes in the kidneys were observed under the microscope after H\&E, PASM, PAS, and Masson's trichrome staining. A proportional area of fibrosis and glomerulosclerosis index were calculated in sections stained with PASM, PAS, and Masson's trichrome. There were no significant pathological changes in the control group. Compared with the control group, the DN group showed glomerular hypertrophy, capillary narrowing, Bowman capsule narrowing, extracellular matrix increasing in the mesangium region, and glycogen and collagen deposition. The pathological injuries to the kidney were more developed in the NBAE group compared to the STZ group $(P<0.05)$. Results are shown in Figure 1.

\section{Electron microscope observations}

Under the electron microscope, the kidney structure was normal in the control group. The glomerular basement membrane was uniform and without thickening, and the distribution of the epithelial foot processes was uniform. In the DN group, the glomerular basement membrane of the rat kidney exhibited significant thickening and was not uniform, having the shape of a hump. Part of the foot processes significantly merged with secondary foot processes, and there was some vascular endothelial cell proliferation. In the NBAE group, the rat kidney showed segmental thickening and foot processes appeared to significantly merge, with different degrees of development. The results are shown in Figure 2.

\section{Detection of expression of TGF- $\beta 1$, collagen IV, and connective tissue growth factor (CTGF) in rat kidney tissues by immunohistochemistry}

Oxidative stress could induce the secretion of cytokines and synthesis and deposition of extracellular matrix by activating TGF- $\beta 1$, collagen IV, and CTGF signal pathways, leading to glomerulosclerosis. There were no significant differences in TGF- $\beta 1$, collagen IV, and CTGF levels in the control group. Compared with the control group, the TGF- $\beta 1$, collagen IV, and CTGF levels were significantly increased in the DN group. Compared with the DN group, TGF- $\beta 1$, collagen IV, and CTGF levels were significantly decreased in the NBAE group $(P<0.05)$. The results are shown in Figure 3. 


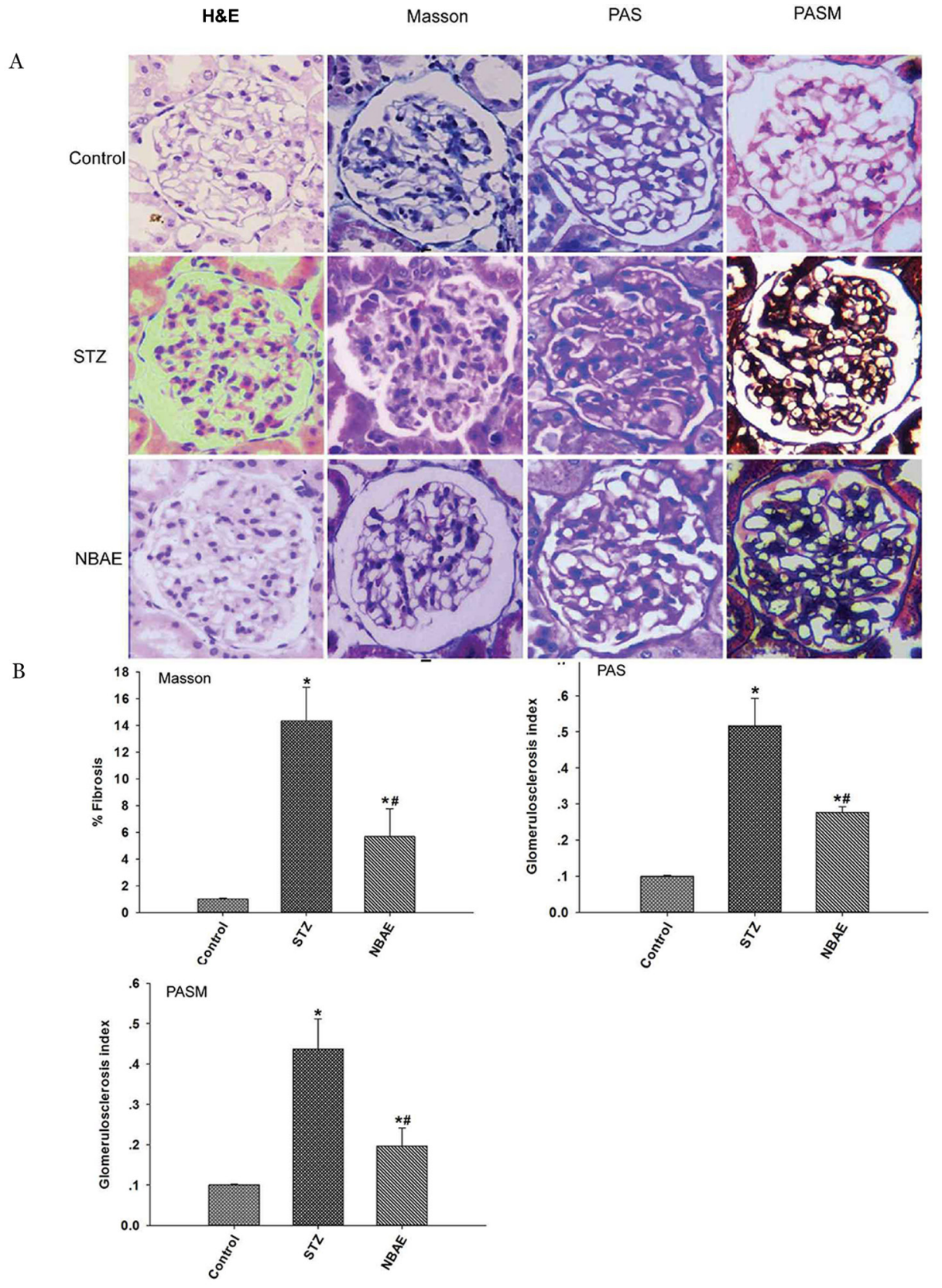

Figure 1. Histopathological changes in the kidneys of diabetic nephropathy (DN) rats with NBAE treatment. A. Kidney tissue was stained with H\&E, Masson's trichrome, periodic acid-Schiff (PAS), and periodic acid-silver metheramine (PASM). Each image is a representative of eight kidney tissue sections in each group. Original magnification: 400X. B. Proportional area of fibrosis and glomerulosclerosis index for various groups as indicated. Masson's trichrome, PAS, and PASM staining were determined semiquantitatively as described above. Data are reported as means $\pm \mathrm{SE}(\mathrm{N}=$ 8). ${ }^{*} \mathrm{P}<0.05$ vs control, ${ }^{\mathrm{P}}<0.05$ vs STZ. 


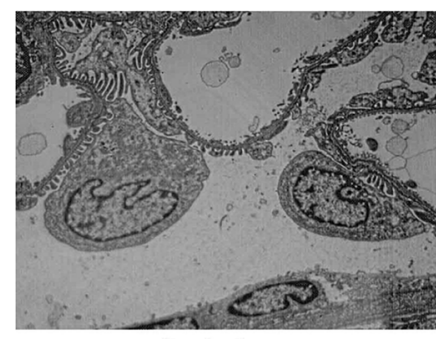

Control

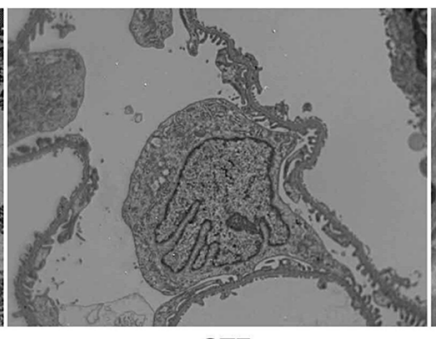

STZ

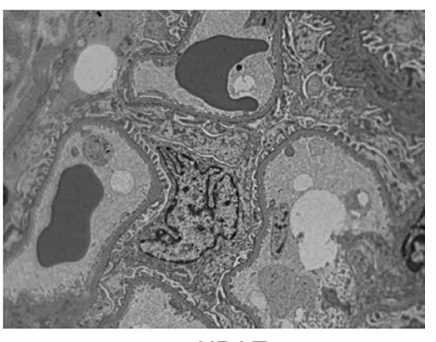

NBAE

Figure 2. Structural changes observed in the kidneys of DN rats by transmission electron microscope (7000X).

A

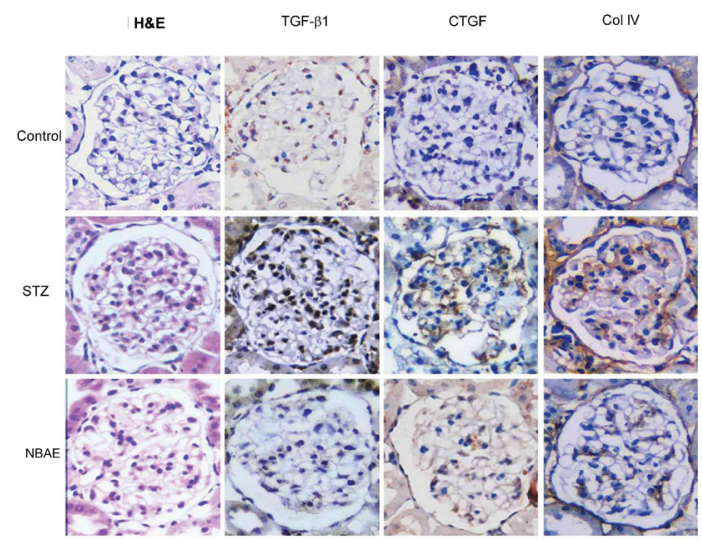

B
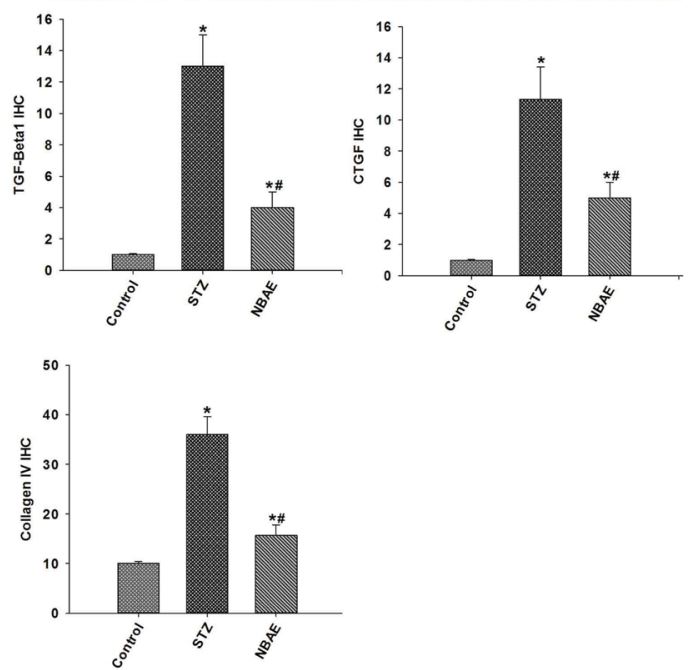

Figure 3. Expression of TGF- $\beta 1$, connective tissue growth factor (CTGF), and collagen IV (Col IV) in kidneys of diabetic nephropathy (DN) rats with NBAE treatment. A. Immunohistochemical photograph of TGF- $\beta 1$, CTGF, and collagen IV in different groups. Original magnification: 400X. B. Semiquantitative analysis of TGF- $\beta 1$, CTGF, and collagen IV immunostaining in different groups as indicated. Data are reported as means $\pm \mathrm{SE}(\mathrm{N}=8),{ }^{*} \mathrm{P}<0.05 \mathrm{vs}$ control, " P $<0.05$ vs STZ. 


\section{DISCUSSION}

DN is a microvessel complication that occurs as a result of DM, and its disease mechanism is complex (Lv and Wang, 2006; Lin et al., 2014). Oxidative stress could activate the pathological pathway related to DM complications, which is a key link in the DN disease mechanism. Traditional Chinese Medical Science has been successfully used to prevent the incidence and development of DN (Hu and Liang, 2013). Further knowledge of the roles of oxidative stress in the DN disease mechanism could provide the framework for preventing and controlling DN.

Rats were fed a high fat diet initially and then injected with STZ after inducing insulin resistance to destroy the islet tissue, resulting in the rat DM model. Rats were continuously fed high energy food to create a rat DN model with constant high blood glucose and proteinuria.

The pathological changes in the early period of DN in the rats were as follows: glomerular hypertrophy and glomerula with high stress, high infusion, high filtration, and proteinuria (Wu et al., 2014). The NBAE decreased the 24-h urine amount, urine protein, HbAlc, BUN, and Scr in DN rats and regulated the glucose metabolism of DN rats, decreasing the discharging of urine protein and developing the kidney function.

Radicals in the DN rat body could attack the polyunsaturated fatty acids in the membranes and induce the over-oxidation of lipid, producing MDA lipid over-oxidation. Oxide and antioxidant imbalance plays an important role in DN (Susztak et al., 2006). NBAE could improve the activities of SOD, CAT, and GSH-Px and total antioxidant ability affecting the oxidation stress of DN rats.

TGF- $\beta 1$ was an important fibrogenic factor and could increase the synthesis of extracellular matrix (ECM). TGF- $\beta 1$ could promote cell proliferation, the production of ECM, and tissue fibrosis and mediate the kidney fibrosis process in DN. CTGF could promote the proliferation of fibroblasts, the production and fibrosis of ECM, and the production of collagen by kidney fibroblasts (Zhao et al., 2010; Fang et al., 2013; Liu et al., 2009, 2014). NBAE could relieve kidney disease in DN rats and cause pathological changes.

NBAE decreased oxidation stress and the expression of TGF- $\beta 1$, CTGF, and collagen IV. It also reduced the degree of fibrosis and hardening index of the glomerulus. The DN rat kidney was improved, suggesting that NBAE had anti-oxidative properties and significant protective effects in DN rats. This study provided evidence that Toona sinensis seeds can be used to prevent DN, and further studies are needed to investigate what specific components play roles in controlling DN and their mechanisms.

\section{Conflicts of interest}

The authors declare no conflict of interest.

\section{ACKNOWLEDGMENTS}

Research supported by the National Natural Science Foundation of China (Grant \#81274049).

\section{REFERENCES}

Du CH, Yan Y, Song Q, Guan AP, et al. (2011). Preliminary study of hypoglycemic effect of aqueous extract from Fructus Toonae sinensis. J. Shanxi Coll. Trad. Chin. Med. 17: 169-171. 
Fang $\mathrm{CH}$, Cheng SH and Wu Q (2013). Effect of Danzhi Jiangtang capsule on NF-кB and urinary albumin excretion rate in early diabetic nephropathy patients. World Sci. Technol.-. Modernization Trad. Chin. Med. 15: 891-895.

Hu J and Liang B (2013). Detection of Serum glucose, uric acid and serum fats in T2DM. China Pract. Med. 8: 138-139.

Hu WS, Liu F and Wang J (2011). Reno-protective effects of curcuma wenyujin on diabetic nephropathy in rats. Chin. J. Exp. Trad. Med. Formulae 17: 163-165.

Jin GL and Chen C (2011). Experimental study on anticoagulation activity of n-butanol extract of Toona sinensis seeds. Chin. J. Hosp. Pharm. 31: 913-914.

Li J and Chen C (2009). Antithrombotic effect of n-butanol extract of Toona sinensis seeds and its mechanisms. Si Chuan Zhon Yi 27: 26-61.

Li YH and Chen C (2010). Effects of total polyphenols extracted from Toona sinensis seeds on the cardiogram and hemodynamics of myocardium ischemia reperfusion rats. J. Anhui Agric. Sci. 38: 15540-15541.

Lin ZT, Zhang C and Shen XM (2014). Advances in pathogenetic mechanisms of diabetic nephropathy. Chin. J. Pharmacol. Toxicol. 28: 765-773.

Liu RR, Zhao TT and Li P (2014). Experimental research progress on therapeutic principle of replenishing Qi, nourishing Yin and activating blood circulation in diabetic nephropathy treatment. World Sci. Technol.-. Modernization Trad. Chin. Med. 16: 1927-1931.

Liu ZH, Dong JX, Xie Y, Hu J, et al. (2009). Effect of mycophenolate on expression of TGF- $\beta 1$ and CTGF in kidney of diabetic nephropathy rats. Chin. J. Hemorheol. 19: 184-188.

Lv Y and Wang YP (2006). Effect of Liuwei Dihuang pill on kidney function of patients with diabetic nephropathy. World Sci. Technol.-. Modernization Trad. Chin. Med. Materiamedica 8: 109-112.

Satirapoj B (2012). Nephropathy in diabetes. Adv. Exp. Med. Biol. 771: 107-122.

Susztak K, Raff AC, Schiffer M and Böttinger EP (2006). Glucose-induced reactive oxygen species cause apoptosis of podocytes and podocyte depletion at the onset of diabetic nephropathy. Diabetes 55: 225-233. http://dx.doi.org/10.2337/ diabetes.55.01.06.db05-0894

Tang Q, Hu H, Wang QS, Fan H, et al. (2010). Effects of Liuwei Dihuang Jiawei Capsula on renal protein kinase C activity and connective tissue growth factor of diabetic nephropathy rats. Chin. Tradit. Herbal Drugs 41: 77-81.

Wu DM, Chen J, Liu JT and Li W (2014). Clinical study on effect of related factors of microalbuminuria in early stage of diabetic nephropathy. World Sci. Technol.-. Modernization Trad. Chin. Med. 16: 2411-2414.

Xing SS and Chen C (2010). Study on the antioxidation of polyphenols from the seeds of Toona sinensis (A.Juss) Roem in vitro. J. Anhui Agric. Sci. 38: 7285-7287.

Yang JW and Li LS (1993). Effects of Rheum on renal hypertrophy and hyperfiltration of experimental diabetes in rat. Zhongguo Zhong Xi Yi Jie He Za Zhi 13: 286-288, 261-262.

Zhao Q, Sun QY and Yang QX (2011). Effects of an anticomplementary polyphenol from the seed of Toona sinensis on complement-injured SH-SY5Y cells. Chin. Pharmacol. Bull. 27: 1086-1090.

Zhao ZJ, Dou XN, Zhang XX and Yang MJ (2010). Effects of Tangshenping capsules on the diabetic nephropathy rats and the expression of TGF- $\beta 1$ protein and mRNA. World Sci. Technol.-. Modernization Trad. Chin. Med. 12: $759-763$. 